\title{
Spatial Influences on Team Awareness and Communication in Two Outpatient Clinics: a Multiple Methods Study
}

\author{
Lisa Lim, PhD' ${ }^{7}$, Matthew Moore, MFA' ${ }^{2}$, Jennifer R. DuBose, MS ${ }^{3}$, Bushra Obeidat, PhD 4 , \\ Robert Stroebel, $M D^{5}$, and Craig M. Zimring, $P h D^{3}$
}

${ }^{1}$ College of Architecture, , Texas Tech University, Lubbock, TX, USA; ${ }^{2}$ Center for Innovation, , Mayo Clinic, Rochester, MN, USA; ${ }^{3}$ School of Architecture, College of Design, , Georgia Institute of Technology, Atlanta, GA, USA; ${ }^{4}$ College of Architecture and Design, , Jordan University of Science and Technology, Irbid, Jordan; ${ }^{5}$ Community Internal Medicine, , Mayo Clinic, Rochester, MN, USA.

\begin{abstract}
BACKGROUND: Healthcare organizations are moving their primary care teams out of private offices into shared workspaces for many reasons, including teamwork improvement and cost reduction.

OBJECTIVE: Identify the specific aspects of layout and design that enable two fundamental processes of highfunctioning teams: communication and situation awareness.
\end{abstract}

DESIGN: This was a multi-method study employing qualitative interviews, floor plan analysis, observations, behavior mapping, and surveys.

PARTICIPANTS: Two primary care clinics in a large, integrated healthcare system in the upper Midwest, with Clinic $\mathrm{S}$ in a suburban location and Clinic $\mathrm{A}$ in a rural setting. In the two clinics, a total of 36 staff members were interviewed, 57 (66\% response rate) staff members were surveyed, and 2013 individual-points were recorded during 63 behavior mapping observations.

MAIN MEASURES: Communication encounters, team members' perception of the environment and teamwork, visibility, distance, functional pathways, and selfreported mode and frequency of staff communication.

KEY RESULTS: Observations, interviews, and surveys identified environmental factors that predict staff awareness and communication patterns. Visibility impacts situation awareness. Frequency of face-to-face communication increases with visibility and proximity between workstations (e.g., Clinic A nurses' intra-role communication without workstation proximity vs inter-role communication with workstation proximity: $22.6 \%$ [11.4, 33.9] vs $77.4 \%$ [66.1, 88.6], $p=0.001$ ) and with staff members' functional paths. Visual exposure to patients predicts staff's concerns about their communication (Clinic S: $2.29 \pm 0.81$ vs Clinic A: $3.20 \pm 0.84, p<0.001)$.

CONCLUSIONS: Design and layout of team spaces have important influences on the way that team members work together. The organizational goals of the healthcare system, particularly which staff members need to work

Electronic supplementary material The online version of this article (https://doi.org/10.1007/s11606-020-05790-6) contains supplementary material which is available to authorized users.

Received November 2, 2018

Revised May 31, 2019

Accepted February 19, 2020

Published online May 4, 2020 together most frequently, should drive the specific design solution.

KEY WORDS: primary care; communication; ambulatory care; teamwork; clinic design.

J Gen Intern Med 35(7):1987-96

DOI: $10.1007 / \mathrm{s} 11606-020-05790-6$

(C) Society of General Internal Medicine 2020

\section{INTRODUCTION}

Many healthcare organizations find that improved teamwork of primary care medical teams improves patient outcomes, ${ }^{1-7}$ quality of care, ${ }^{1,2,4}$ patient satisfaction, ${ }^{1,7}$ and employee satisfaction. ${ }^{1}$ To improve teamwork, healthcare organizations are redesigning the physical space of their primary care clinics, moving providers out of their offices to multidisciplinary team rooms where they are co-located with other providers and with staff such as nurses and medical assistants. ${ }^{8,9}$ Co-location is just one factor of teamwork, ${ }^{10}$ and its role is conflicting in previous studies: while there is evidence that co-location can increase teamwork of clinical members, ${ }^{11,}{ }^{12}$ other recent studies, especially in non-clinical work settings, have found that co-location is associated with decreased communication. ${ }^{13,14}$ This paper explores primary care teamwork in physical space to clarify physical design strategies that can support better teamwork.

A recent review of the interprofessional collaborative practice literature found that spaces for interprofessional primary care teams need to support frequent communication by allowing team members to see each other and be near each other, while still providing opportunities for heads-down individual work. ${ }^{15}$ Visibility to co-workers not only facilitates spontaneous and intentional communication but also contributes to greater awareness of peers, greater situation awareness, and increased opportunities for knowledge sharing and tacit learning. ${ }^{16}$ A recently published systematic literature review reported that visibility and accessibility were among the most frequently cited specific design factors contributing to teamwork and communication in healthcare environments. ${ }^{17}$ 
The purpose of this study is to identify key spatial metrics that define co-location and affect two fundamental processes of teamwork - communication and situation awareness-using the conceptual model shown in Figure 1. These spatial attributes can be defined and measured to provide a useful tool to aid decisions during clinic development and design.

\section{METHODS}

\section{Study Design and Oversight}

This multi-method study employed interviews, spatial analysis, observations, and surveys in two clinics. The research protocol was reviewed and approved by the Georgia Institute of Technology and Mayo Clinic Institutional Review Boards. Participants received and were asked to review a written consent document with information about the study, but documentation of consent was not required for the minimal risk research.

\section{Settings}

The two Mayo Clinic primary care clinics were chosen since both clinics have team-based architectural layouts identified in the organization's building standards document and had highperforming teams as indicated by Team Development Measurement ${ }^{18}$ scores collected internally for certification of the system's community care model (Fig. 2). At the core of the system's care model is the optimized care team - a co-located, interprofessional team of clinicians, nurses, allied health staff, and other specialized staff. Clinic A is a small rural clinic, newly built in 2016, with a panel size of 4000. The Clinic A staff operates as a single team with three provider/nurse subteams with shared RN care coordination, RN triage, interpreter, and behavioral health members. Telehealth connections to regional specialists are routinely used. Clinic $\mathrm{S}$ is a larger urban/suburban clinic comprised of two large teams, each with three to four providers, supported by a range of shared services similar to Clinic A's and a panel size of 15,000. Clinic S's team space was renovated in 2016 to provide visual connections among staff members.

The primary differences include the following. Clinic A's team workspace is adjacent and open to patient circulation; a central enclosed team huddle space visually separates the subteams. Clinic S's team space is a large central room separate from patient circulation with clear sightlines across the space. Clinic A's providers and their dedicated nurse and assistant teammates sit adjacent to one another. In Clinic S, staff workstations are clustered by roles (e.g., nurses sit next to nurses), but team members of other roles are visible. Lastly, Clinic S has significantly more non-visit care activity, such as responding to phone calls or patient messages via online portal, than Clinic A.

\section{Data Collection Procedure}

Architectural floorplans and clinic staff data were obtained prior to visiting the sites. A preliminary site visit (September 20-22, 2017) was conducted, followed by a more in-depth site visit (November 13-17, 2017) to conduct face-to-face interviews, surveys, and observations. Both site visits were conducted by two or three members from the Georgia Tech research team. The clinic administration distributed online social network surveys to the staff

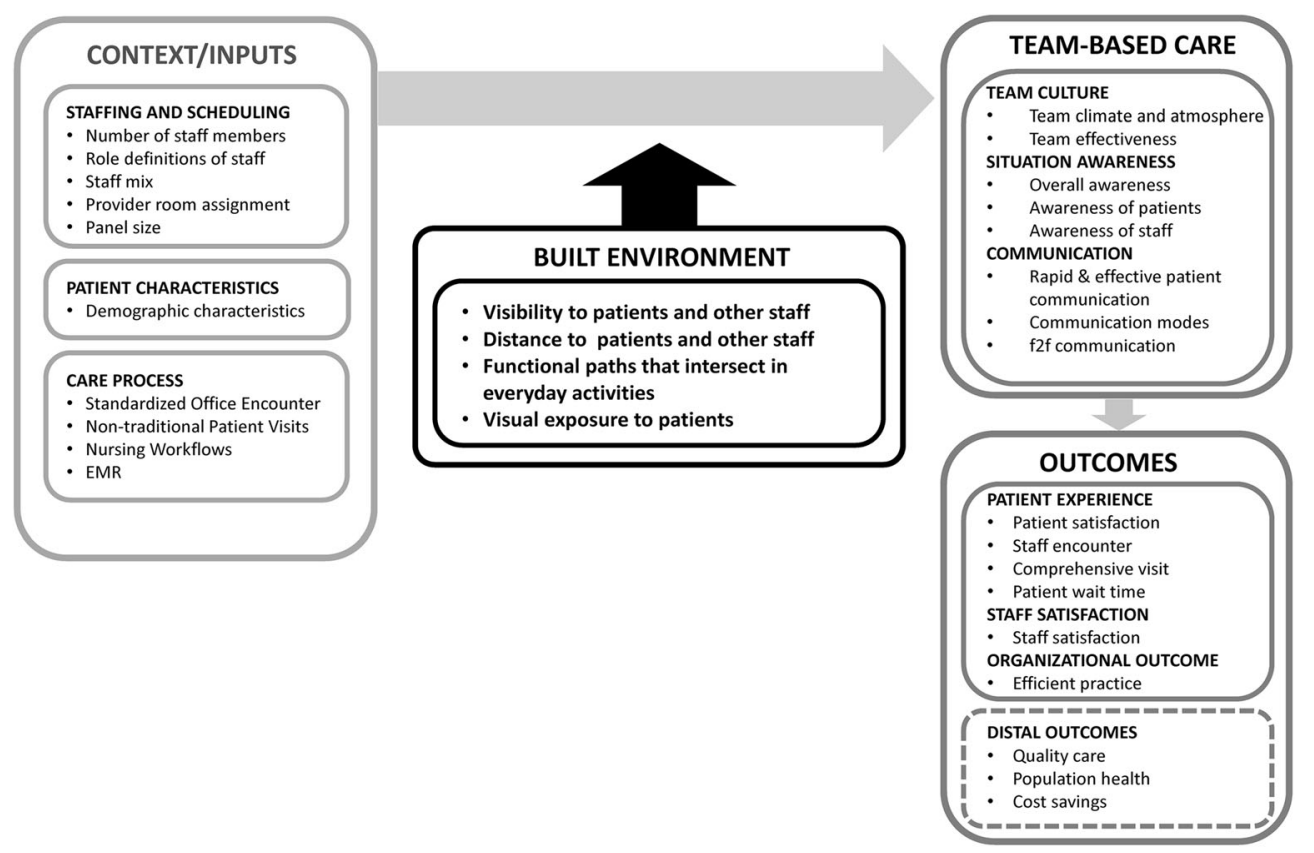

Figure 1 The built environment moderates the relationship between care process, process variables, and outcomes. 
EXAM ROOM

TEAM AREA

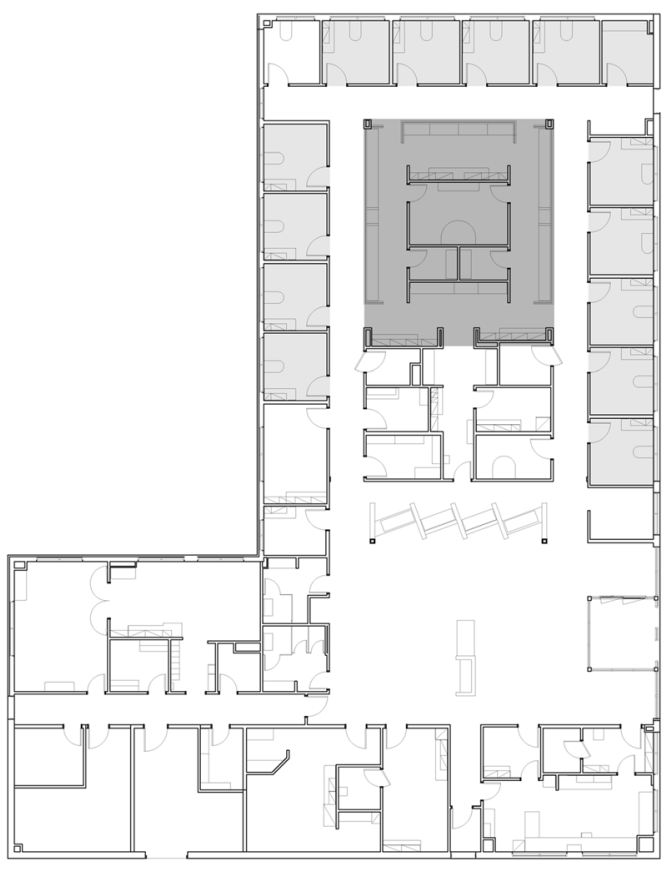

CLINIC A

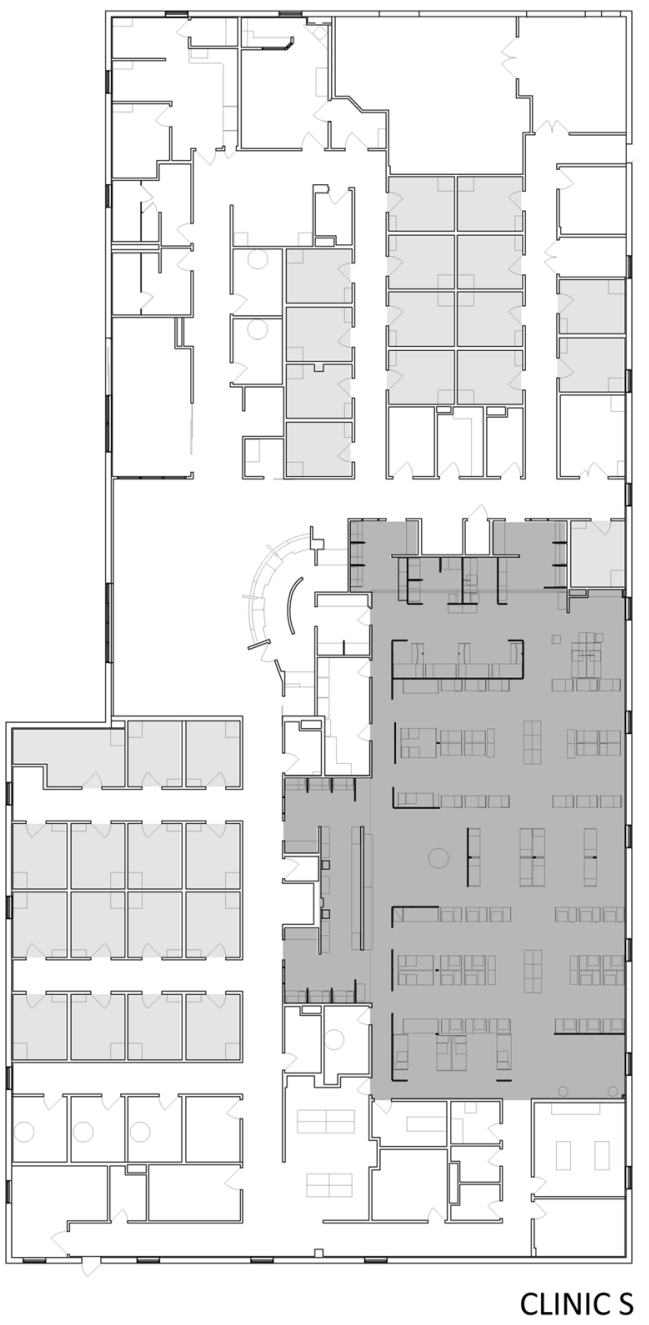

Figure 2 Layouts of the two clinics (left: Clinic A, right: Clinic S). Clinic A's team workspace is adjacent and open to patient circulation, and Clinic S's team space is a large central room separate from patient circulation and the exam rooms.

electronically. Other than online survey distribution, no members of Mayo Clinic participated in the data collection procedure, so as not to bias data outcomes.

\section{Spatial Analysis}

To quantify spatial properties of the two clinics, spatial analysis was performed using multiple programs. Physical distances were calculated in AutoCAD and ArcGIS programs, and visual relationships between staff members (i.e., number of other staff workstations visible from each workstation) were analyzed using the VisualPower tool. ${ }^{19}$

\section{Teamwork Outcome Measurements}

The study used several quantitative and qualitative methods to collect staff teamwork data including qualitative observations, interviews, behavior mapping observations, and surveys.

Qualitative Observations and Interviews. Qualitative field observations and semi-structured interviews were conducted to identify the spatial qualities of the settings and evaluate team members' perceptions of the environment and teamwork.
At least one staff member representing each role in the clinic was interviewed to get a well-rounded understanding of each perspective and to provide contextual information regarding the care process, operational culture, and staff experience in the current layouts (see online appendix for interview questions).

Behavior Mapping Observations. Behavior mapping observations were conducted in the clinics for both morning and afternoon sessions to document team members' encounters and activities. Researchers made repeated circuits of the clinics, noting the locations of staff members and patients and their activities (see online appendix for protocols). The team used a proprietary tablet-based application to record encounters and locate them in the architectural layout. $^{20}$

Staff Teamwork Survey. Staff members' perception regarding awareness and concerns for having patient-related communication were collected using a paper survey developed for the study (see online appendix for the survey items). The survey 
employed a 5-point Likert scale. Though Likert scales are ordinal scales, this study used the average of all items as the composite score for each survey construct in order to compare the composite scores of the two clinics. All available staff members during the data collection visits were asked to participate.

Staff Social Network Analysis Online Survey. A social network questionnaire was developed to map communication relationships of staff members (see online appendix for the questionnaire). The survey included three types of communication (routine work, requesting advice, and personal interest) and two modes of communication (face-to-face communication (F2F) and virtual communication $(\mathrm{V}))$. For each type and mode of communication (e.g., routine work F2F, routine work $\mathrm{V}$ ), participants were asked to identify their communication counterparts and the frequency. The online survey was administered during and after the data collection visits, and staff members were reminded to answer the survey multiple times.

\section{Statistical Analysis}

Statistical analysis was performed using SPSS 24. Descriptive statistics were conducted to determine means and standard deviations. $T$ test, analysis of variance (ANOVA), and chisquare tests were used to examine group differences.

\section{RESULTS}

\section{Data Collection Summary}

In two clinics, a total of 36 individuals were interviewed, 64 behavior mapping rounds were recorded, 57 (response rate, $66 \%$ ) staff members completed the paper survey, and 24 (response rate, 22\%) staff members answered the social network survey (Tables 1 and 2).

\section{Environmental Factors Associated with Awareness}

Qualitative observations, interviews, and survey comments identified three environmental factors that influenced staff members' awareness: visibility of team members determined by internal layout of team area, visibility of overall clinic area from team areas, and accessibility and visibility of exam rooms from team rooms (Table 3 ).

Visibility among Staff Members and Mutual Awareness. The average visibility of all other workstations from each individual workstation was significantly lower in Clinic A $28.6 \%(5.7 / 20)$ compared with the average of $53.0 \%$ (27.5/ 52 ) in Clinic $\mathrm{S}$ due to the visual obstruction from the huddle room in the middle of the team area (Table 1). However, the survey results reported that the staff of both clinics had good situation awareness of other team members. The team areas in both clinics supported staff members' ability to find each other when needed, to approach each other when requesting or offering advice/help, and to be aware of other team members' needs. No statistically significant differences between the two clinics were found (Table 1).

Some specific roles had low visibility between team members due to layout. In Clinic A, staff members felt they were "separated" into two groups. A staff member stated in the survey, "There are days I don't ever see those on the other side." This low visibility particularly seemed to impact those who share workloads. LPNs in Clinic A, taking care of nurse-only-visit patients collectively, had to develop a "complex" way of communicating electronically to compensate for the lack of visibility to each other due to the location of huddle room. Similarly, a provider in Clinic A described his experience of moving into the

Table 1 Descriptive and Statistical Test Results of Visibility, Staff Interviews, and Surveys

\begin{tabular}{|c|c|c|c|c|}
\hline \multirow[t]{2}{*}{ Approach } & \multirow[t]{2}{*}{ Clinic A } & \multirow[t]{2}{*}{ Clinic S } & \multicolumn{2}{|c|}{ Comparison between the two clinics ( $t$ test) } \\
\hline & & & Mean difference ( $p$ value) & $95 \%$ CI $[\mathbf{L L}, \mathbf{U L}] *$ \\
\hline \multicolumn{5}{|l|}{ Workstation visibility } \\
\hline Total number of workstations $(N)$ & 20 & 52 & & \\
\hline Mean number of visible other workstations & 5.7 & 27.5 & & \\
\hline Mean ratio of workstation visibility & $28.6 \%$ & $53.0 \%$ & $-24.4\left(p<0.001^{\dagger}\right)$ & {$[-35.3,-13.5]$} \\
\hline \multicolumn{5}{|l|}{ Interviews } \\
\hline Participants & $11^{*}$ & $25^{\S}$ & & \\
\hline \multicolumn{5}{|l|}{ Staff survey } \\
\hline Available staff members during the visits & 24 & 63 & & \\
\hline Participants $(N)$ & 20 & 37 & & \\
\hline Response rate & $83 \%$ & $59 \%$ & & \\
\hline Awareness (4 items): Mean (SD) & $4.15(0.57)$ & $4.34(0.59)$ & $-0.19(p=0.25)$ & {$[-0.51,0.14]$} \\
\hline Communication concerns (4 items): Mean (SD) & $3.20(0.84)$ & $2.29(0.81)$ & $0.91(p<0.001)$ & {$[0.46,1.37]$} \\
\hline
\end{tabular}

* LL and UL indicate the lower-level limit and upper-level limit of a confidence interval, respectively

+ The number of degrees of freedom was adjusted since Levene's Test for Equality of Variances was statistically significant

+3 providers, 2 licensed practical nurses, 1 registered nurse, 1 behavioral health specialist, 1 receptionist, 2 administrative staff, and 1 interpreter

$\$ 2$ providers, 9 licensed practical nurses, 1 registered nurse, 2 care coordinators, 1 social worker, 1 behavioral health specialist, 2 administrative staff, 4 clinical assistants, 2 patient appointment coordinators, and 1 phone triage nurse

"Survey items used a 5-point Likert scale (strongly disagree to strongly agree). A higher score indicated better awareness and higher concerns for talking about patients 
Table 2 Communication Pattern Results from Behavior Mapping Observation and Social Network Survey

\begin{tabular}{|c|c|c|}
\hline Approach & Clinic A & Clinic S \\
\hline \multicolumn{3}{|l|}{ Behavior mapping observation } \\
\hline $\begin{array}{l}\text { Observation rounds (interval between } \\
\text { rounds) }\end{array}$ & $37(15 \mathrm{~min})$ & $26(30 \mathrm{~min})$ \\
\hline Data points* & 667 & 1346 \\
\hline Data length & $9.25 \mathrm{~h}$ & $13 \mathrm{~h}$ \\
\hline \multicolumn{3}{|l|}{ Social network survey $^{\dagger}$} \\
\hline$N$ (response rate, total staff members) & $6(19 \%, 32)$ & $18(23 \%, 78)$ \\
\hline \multicolumn{3}{|c|}{ Face-to-face "routine work" communication } \\
\hline $\begin{array}{l}\text { Data points } \\
\text { ANOVA test for mean distances per } \\
\text { communication frequency significance }\end{array}$ & $\begin{array}{l}121 \\
p<0.001^{\|}\end{array}$ & $\begin{array}{l}717 \\
p<0.001^{\|}\end{array}$ \\
\hline \multicolumn{3}{|l|}{ Virtual "routine work" communication } \\
\hline Data points & 101 & 611 \\
\hline $\begin{array}{l}\text { ANOVA test for mean distances per } \\
\text { communication frequency significance }\end{array}$ & $p=0.23$ & $p<0.001^{\| \prime}$ \\
\hline \multicolumn{3}{|c|}{ Face-to-face "seeking advice" communication } \\
\hline Data points & & 214 \\
\hline $\begin{array}{l}\text { ANOVA test for mean distances per } \\
\text { communication frequency significance }\end{array}$ & $p=0.04$ & $p<0.001$ \\
\hline \multicolumn{3}{|l|}{ Virtual "seeking advice" communication } \\
\hline $\begin{array}{l}\text { Data points } \\
\text { ANOVA test for mean distances per } \\
\text { communication frequency significance }\end{array}$ & $\begin{array}{l}31 \\
p=0.004^{\|}\end{array}$ & $\begin{array}{l}177 \\
p<0.001^{11}\end{array}$ \\
\hline
\end{tabular}

* Each data point represents a single individual

\% The social network survey responses were translated into communication relationships for each type of communication. The low response rate for the personal interest communication type provided too few data points to include in the analysis

$\neq$ Each data point represents communication frequency between two individuals

\$ Mean distances between staff members per communication frequency and post hoc Tukey HSD multiple comparison results are illustrated in Fig. 4

"An adjusted F test was performed using Brown-Forsythe statistic since an assumption of homogeneity of variance was not met

new clinic layout. The new layout of Clinic A had a shared team area, but the previous layout had separate, distant workspaces for providers and LPNs. The provider stated that in the previous layout, he used to spend 5-10\% of his time looking for nurses, compared to the current $3 \%$.

In Clinic S, providers, RNs, and other shared services were co-located in two main team clusters with good visibility to each other. The LPN workstations located on the edge of the team area, however, were visually segregated from other staff members and other LPN workstations. LPNs were not able to consistently anticipate team needs or fully leverage LPNs from the other workstation. This lack of awareness led to situations illustrated by an exchange shared by an LPN: a receptionist told her, "a patient had been waiting for more than $20 \mathrm{mi}$ nutes," to which she replied, "what are the other LPNs on that side doing?"

Visibility of General Clinic Area and Overall Awareness. Being able to see the general clinic area from team areas seems important to support awareness of the overall clinic for the staff. Absence of this awareness may hinder staff members' ability to quickly and collectively respond to abnormal situations, anticipate possible problems, and react to events that happen elsewhere in the clinic.
In Clinic A, the location of the huddle room reduced staff members' overall awareness since it blocked visibility to the other side of the clinic from their workstations. An RN shared her frustrating experience of not knowing an ambulance had arrived to take a patient in urgent need due to the blocked visibility. The clinic subsequently compensated for the lack of visibility with an alarm system for emergency situations.

An LPN at Clinic S reported a similar experience of not being aware when an emergency situation occurred. Since LPN stations were relatively segregated from the rest of the team area, the LPN did not know there was a patient in urgent need in the waiting area until she heard people running out to assist the patient.

Visibility and Proximity of Exam Rooms from Team Areas and Awareness of Patients. Visibility of exam rooms is important for allowing staff to be aware of patients' status. This awareness was particularly well supported in Clinic A where team workstations faced their assigned exam rooms with only a chest-height glazed workstation partition for separation. Providers and LPNs debriefed in the corridor immediately after exiting the exam room for each patient since they were able to see each other instantaneously. This connection was not only achieved by the layout but also supported by the operational culture of assigning exam rooms for each pod in Clinic A. While staff members did not have visibility of all exam rooms, they had direct visibility of their assigned exam rooms. One trade-off was that adjacency of the hall to staff stations had raised some concern for patient privacy.

In comparison, the exam rooms in Clinic $\mathrm{S}$ were only visible from the LPN stations. Even staff at the LPN stations had a hard time seeing their two assigned corridors, as each station had visibility of only one patient corridor. This lack of direct visibility from their stations was mitigated by an electronic room monitoring system showing room assignments and occupancy, which staff frequently checked from their workstations.

\section{Environmental Factors Associated with Communication}

In addition to awareness, this study investigated how the layout of clinics impacts face-to-face communication patterns of staff members. Our analysis identified four main spatial metrics: visibility, distance, functional paths, and visual exposure to patients (Table 3).

Visibility and Proximity among Staff Members and Communication Frequency. Visibility among staff members enabled by co-location facilitated frequent face-to-face communication. A care coordinator working in two different clinics (Clinic S and Clinic C) in the same organization shared her experiences of different communication patterns due to the clinic layouts. She stated that she used electronic messages for $90 \%$ of her communication with providers in Clinic $\mathrm{C}$ where providers had individual offices. She communicated 
Table 3 Summary of Design Strategies and Related Teamwork Aspects

\begin{tabular}{|c|c|c|c|}
\hline Design categories & Clinics & Utilized design strategy & Related teamwork outcomes \\
\hline \multicolumn{4}{|c|}{ Clinic layout and team area attributes } \\
\hline \multirow[t]{2}{*}{$\begin{array}{l}\text { Team area locations } \\
\text { in the clinic }\end{array}$} & $\begin{array}{l}\text { Clinic } \\
\text { A }\end{array}$ & $\begin{array}{l}\text { Team area in the middle of the clinic, but } \\
\text { visibility of overall clinic blocked by a huddle } \\
\text { room in team area }\end{array}$ & $\begin{array}{l}\text { - Staff members not knowing activities/instances occurring on the } \\
\text { other side of the clinic from their workstations unless notified } \\
\text { electronically or via alarm system }\end{array}$ \\
\hline & $\begin{array}{l}\text { Clinic } \\
\text { S }\end{array}$ & $\begin{array}{l}\text { Team area in the middle of the clinic, but } \\
\text { clinic area not visible from workstations }\end{array}$ & $\begin{array}{l}\text { - Staff members not knowing activities/instances occurring in } \\
\text { clinic area from their workstations unless notified by } \\
\text { other staff members }\end{array}$ \\
\hline \multirow{2}{*}{$\begin{array}{l}\text { Team area and exam } \\
\text { room relations }\end{array}$} & Clinic & Close proximity with visual connections & - Staff at workstations easily aware of patients in exam rooms \\
\hline & $\begin{array}{l}\text { Clinic } \\
\text { S }\end{array}$ & Separated, electronic system used & $\begin{array}{l}\text { - Staff at workstations mostly used computers (room monitoring } \\
\text { system) to be aware of patients in exam rooms }\end{array}$ \\
\hline \multirow[t]{2}{*}{$\begin{array}{l}\text { Team area visual } \\
\text { exposure to patients }\end{array}$} & Clinic & Exposed & $\begin{array}{l}\text { - Moderate degree of communication privacy concerns (4-item } \\
\text { staff survey, } M=3.20 \text { ) }\end{array}$ \\
\hline & $\begin{array}{l}\text { Clinic } \\
\mathrm{S}\end{array}$ & Enclosed & $\begin{array}{l}\text { - Staff less concerned about having patient-related communica- } \\
\text { tions (4-item staff survey, } M=2.29 \text { ) }\end{array}$ \\
\hline \multicolumn{4}{|c|}{ Internal layout of team areas } \\
\hline \multirow[t]{2}{*}{$\begin{array}{l}\text { Visibility between } \\
\text { staff workstations }\end{array}$} & $\begin{array}{l}\text { Clinic } \\
\text { A }\end{array}$ & $\begin{array}{l}\text { Partially blocked by a huddle room in the } \\
\text { middle (on average, } 28.6 \% \text { of workstations } \\
\text { visible) }\end{array}$ & $\begin{array}{l}\text { - Overall good awareness of team members (4-item staff survey, } \\
M=4.15 \text { ), but lack of awareness between staff members on the } \\
\text { other side of the team area } \\
\text { - Overall frequent staff face-to-face communication }\end{array}$ \\
\hline & $\begin{array}{l}\text { Clinic } \\
\mathrm{S}\end{array}$ & $\begin{array}{l}\text { Workstations visually connected (on average, } \\
53.0 \% \text { of workstations visible), but LPN } \\
\text { stations segregated }\end{array}$ & $\begin{array}{l}\text { - Overall good awareness of team members (4-item staff survey, } \\
M=4.34) \text {, but segregated LPNs less aware of other staff } \\
\text { members } \\
\text { - Overall frequent staff face-to-face communication }\end{array}$ \\
\hline \multirow[t]{2}{*}{$\begin{array}{l}\text { Distance between } \\
\text { workstations }\end{array}$} & $\begin{array}{l}\text { Clinic } \\
\text { A }\end{array}$ & Pod (different role*) adjacency & $\begin{array}{l}\text { - Higher ratio of observed different role* communication } \\
\text { instances (e.g., LPN-Other roles }=77 \% \text { vs. LPN-LPN }=23 \% \text { ) }\end{array}$ \\
\hline & $\begin{array}{l}\text { Clinic } \\
\mathrm{S}\end{array}$ & Role (same role*) adjacency & $\begin{array}{l}\text { - Higher ratio of observed same role*communication instances } \\
\text { (e.g., LPN-Other roles }=13 \% \text { vs. LPN-LPN }=87 \% \text { ) }\end{array}$ \\
\hline \multirow[t]{2}{*}{ Functional paths } & Clinic & No same role* overlap & $\begin{array}{l}\text { No specific same role* communication observed in relation to } \\
\text { functional paths }\end{array}$ \\
\hline & $\begin{array}{l}\text { Clinic } \\
\text { S }\end{array}$ & Different role* overlap & $\begin{array}{l}\text { - Provider-LPN communication observed near LPN stations } \\
\text { (providers talking to LPNs on their way out to exam rooms or } \\
\text { into their workstations) }\end{array}$ \\
\hline
\end{tabular}

* Same role: individuals with same roles (intra-role); different role: individuals with different roles (inter-role)

primarily face-to-face with providers in Clinic S where care coordinators and providers shared an open team area and rarely used electronic messages.

The behavior mapping observations and online survey results demonstrated that shorter distances between workstations were strongly associated with increased staff face-to-face communication frequency (Table 2). In Clinic A, one provider, one $\mathrm{RN}$, and one LPN were assigned to each other as members of a pod and physically clustered together proximate to "their" exam rooms. Conversely, Clinic $\mathrm{S}$ did not assign staff members to a pod; rather, a group of providers, nurses, and other staff formed a larger team with each role generally seated together (e.g., providers sat adjacent to providers). As shown in Figure 3, we observed frequent communication between roles sitting adjacent to each other: inter-role communication was greater in Clinic $\mathrm{A}$, and intra-role communication was greater in Clinic $\mathrm{S}$ (see online appendix for descriptive and statistical test results of the observed communication counterparts).

The reported communication patterns confirmed the association of distance with the face-to-face communication frequency. In both clinics, staff members with shorter average distances reported having more frequent face-to-face communication (for both routine-work and seeking-advice communications). The frequency of virtual communication did not show a strong linear tendency in relation to the distance between workstations according to ANOVA and post hoc Tukey test results (Table 2 and Fig. 4). This pattern suggests that workflow, rather than layout, is the principal driver of virtual communication.

Overlapped Functional Paths of Staff Members and Unplanned Communication. Movements and functional pathways of staff members seemed to impact communication patterns, similar to visibility and distance between workstations. Staff members occasionally talked to other staff members on their functional pathways. For instance, LPNs immediately reported to providers near exam rooms in Clinic A, and providers in Clinic S talked to LPNs near LPN stations on their way to or from exam rooms. In addition, providers in Clinic S stated that the internal layout of their team's workspace enabled them to talk with other team members (RNs or LPNs) on their way to and from exam rooms, encouraging immediate and frequent communication.

Visual Exposure to Patients and Communication Concerns. The level of visual and auditory exposure to patients was significantly different between the two clinics. The team area in Clinic A had visual and auditory accessibility from patient 


\section{Clinic A: Seating by Team}

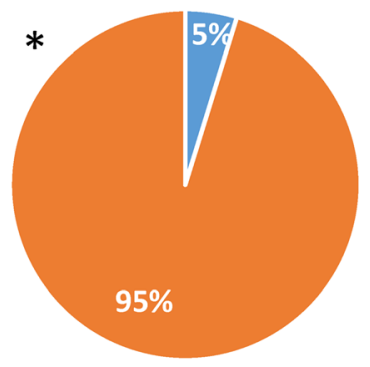

\section{Providers}

\section{Clinic S: Seating by Role}

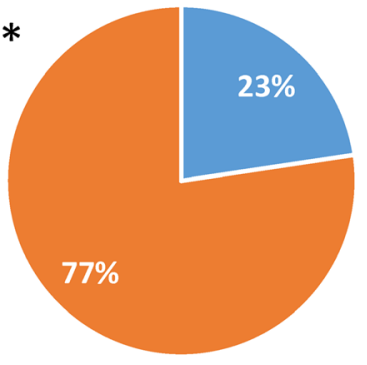

LPNs
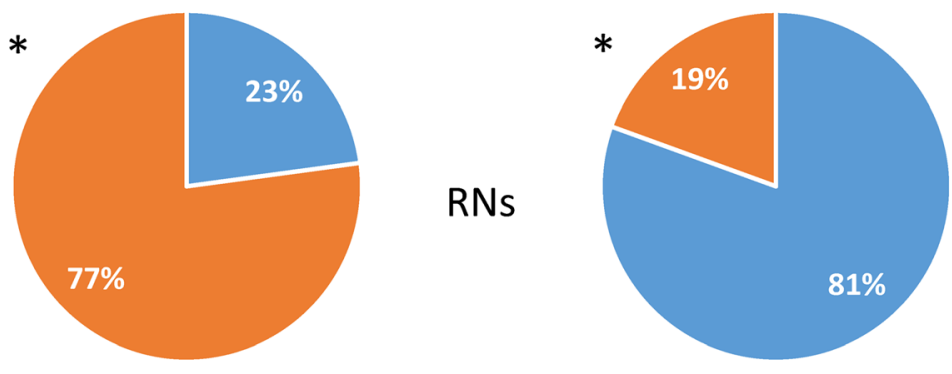

Talking with people of different role

Talking with people of same role
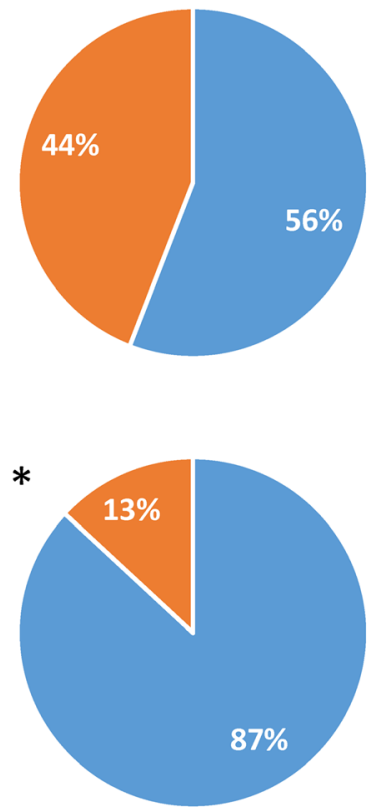

Figure 3 Communication counterparts and frequency from behavior mapping results. Frequent communication between roles sitting adjacent to one another were observed. More inter-role communication was observed in Clinic A, and frequent intra-role communication was observed in Clinic S. (The asterisk means the difference between two percentages, talking with people of different roles and talking with people of same role, is statistically significant at the 0.01 level. See online appendix for descriptive and statistical test results regarding the observed communication counterparts).

corridors. The team and LPN areas in Clinic S were only indirectly visible and sound transmission was very low. These different levels of visual and sound exposure to patients were associated with distinct degrees of concern for privacy when having patient-related communication in the team room. Based upon our staff survey, staff members in Clinic S were less concerned about having patient-related communication in the team area compared to staff members in Clinic A $(2.29 \pm 0.81$ vs $3.20 \pm 0.84, p$ value of $<0.001)$ (Table 1).

The level of concern recorded here is the staff's self-reported perception, and this study did not assess actual patient privacy. The sense of safety created by the closed team area in Clinic S resulted in staff members speaking in a normal volume about patients in areas of the team room. Anecdotally, this led to some auditory exposure to patients in the waiting areas.

\section{DISCUSSION}

The built environments and the related cultures and workflows of the two clinics studied generally facilitated the teams' situation awareness and communication. Visibility (of other team members, clinical area, and exam rooms), proximity of workstations, overlapping functional paths, and visual exposure to patients determined by clinical layout predicted situation awareness and communication (Table 3). These findings correspond to previous studies regarding the role of co-location and visibility, ${ }^{11}, 12,21,22$ and movement of individuals, ${ }^{20}$ in communication and teamwork. Having direct visibility specifically supported the mutual awareness of the activities of those who shared workload. Being aware of overall clinic area and patient exam rooms through direct visibility (or through technology that can substitute for direct visibility) supported team members having a better understanding of the clinic and 

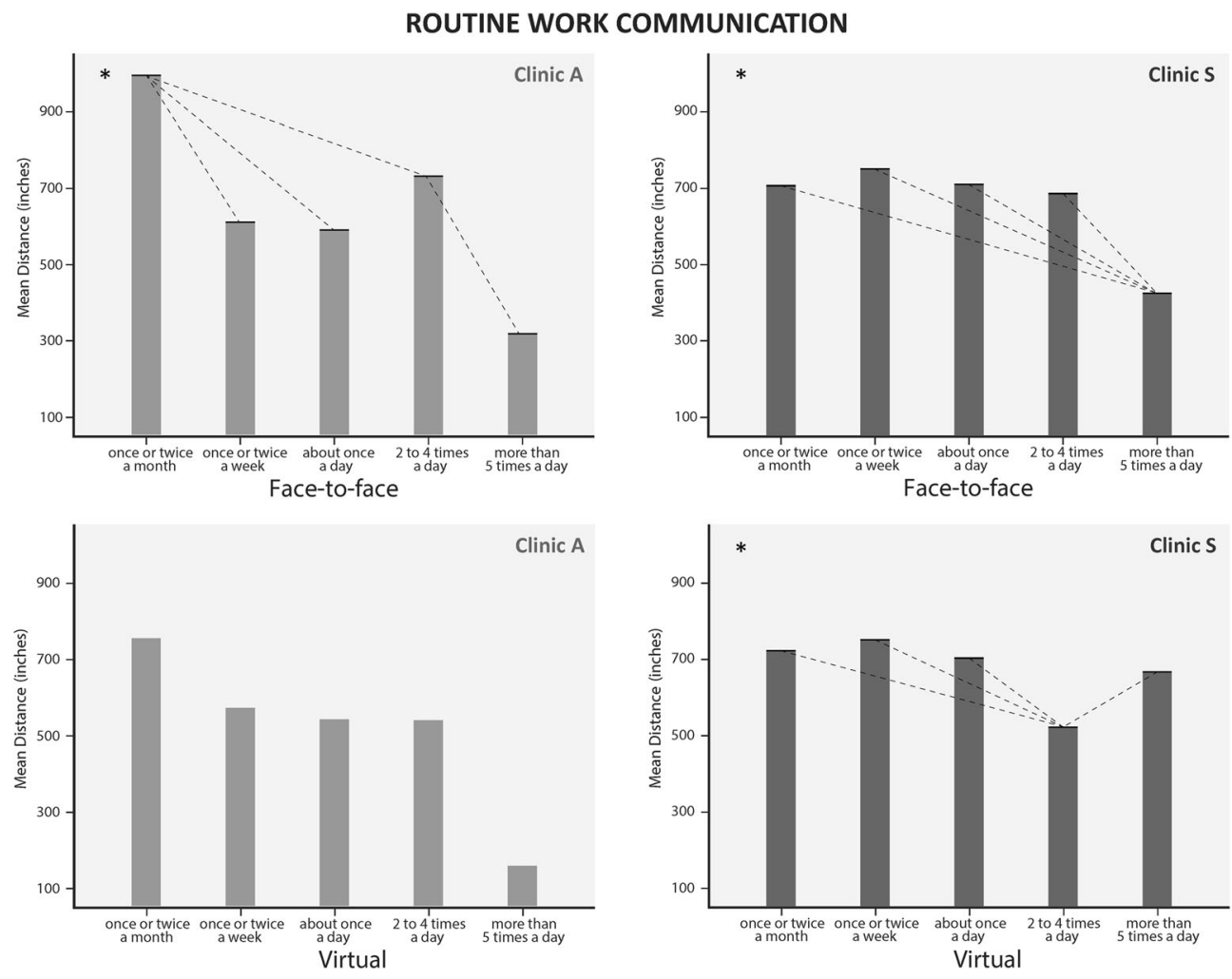

SEEKING ADVICE COMMUNICATION
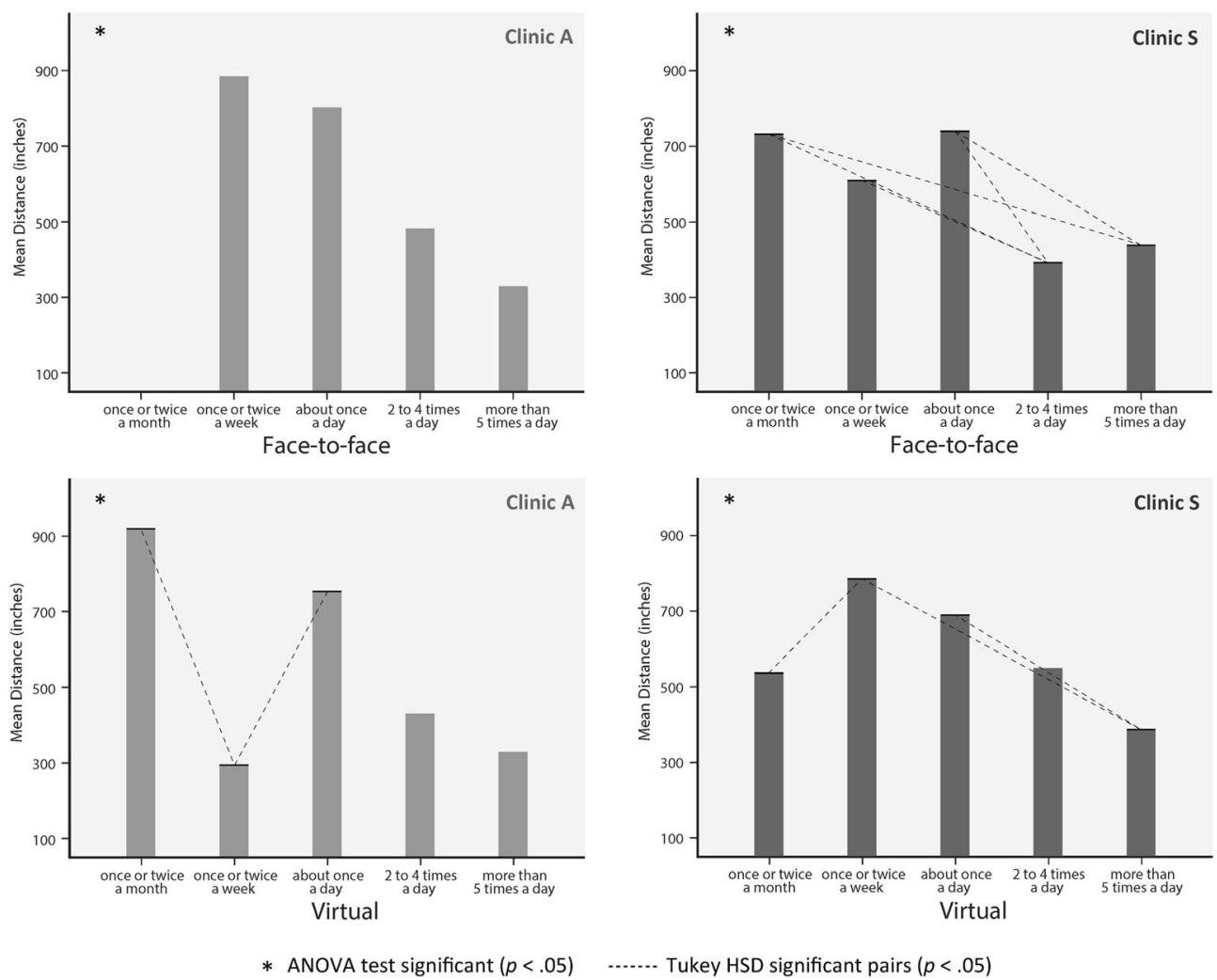

Figure 4 Mean distance between workstations per communication frequency. ANOVA and post hoc Tukey tests revealed that more frequent face-to-face communication occurred between people with shorter average distances. The frequency of virtual communication did not show a strong linear relationship with the distance between workstations.

patients and being able to react to emergent situations. Locating workstations of staff members who needed to talk to each other next to each other increased their communication frequency, as did functional pathway adjacency. 
These findings reflect some of the complexity of teamwork in primary care. Most team activities were routine, but some rare events, such as an ambulance arriving, required immediate mobilization of a larger team spread across separate spaces. While both in-person and electronic encounters were inherently multidisciplinary, there were some apparent differences in patterns of communication. Although in-person patient encounters included a provider and LPN, electronic patient encounters such as email, phone, or video were typically handled by RNs who needed to consult with other RNs frequently. Clinic S had a much higher volume of electronic encounters, which drove the clustering of RNs in proximity to each other rather than distributing them among the teams as in Clinic A. These findings are informative for consultative and primary care internal medicine practices given the increasing reliance on team-based care and electronic communication with patients and colleagues.

For health system leaders with responsibility for new clinic construction or redesign, these findings have significant implications. In a practice evolving toward multidisciplinary, top-of-licensure, team-based care, a premium should be placed on co-location and proximity of key team members. For peripheral team members, visibility or overlapping paths should be considered. Line of sight to patient care areas can enhance situation awareness. While the renovation of an entire clinic may be unattainable due to cost or constraints of existing architecture, reconfiguration of furniture layout and reassignment of workstations can be done in existing spaces to provide visual and physical connections between staff members and to overlap functional paths of staff members.

There are some limitations to this study. Teamwork is multifactorial, and this study does not assess additional factors such as leadership and training. This study found significant associations between physical attributes and communication patterns, but the cross-sectional design of the study does not enable claims of causality. It included only two clinics and did not allow consideration of panel size or exam room efficiency. While different patterns of communication frequency were observed in the two clinics (more frequent intra- or inter-role communication), impact on patient safety or outcome was not examined, and the observations were made during a small window of time at each clinic. Additionally, this study does not examine possible trade-offs of design decisions. For instance, as shown in Clinic S, visual separation may decrease patient privacy concerns but inhibit direct staff visibility to clinic areas and the resulting awareness of patient status. Future studies should include exploring potential technological, operational, cultural, and spatial design strategies to mitigate the negative consequences of these trade-offs.

Acknowledgments: The authors would like to thank Lori Baumbach and Deb Cholewa, operational leaders for the two clinics featured in this paper, and the many staff members of these clinics who generously gave their time and effort to the study. We would also like to thank Aaron Biedermann for his efforts enabling the collaboration between Georgia Tech and Mayo Clinic.
Corresponding Author: Craig M. Zimring, PhD; School of Architecture, College of Design, Georgia Institute of Technology, Atlanta, GA, USA (e-mail: craig.zimring@design.gatech.edu).

Funding Information The project described was supported by Funding Opportunity Number 1L1CMS31449-01 from the U.S. Department of Health \& Human Services, Centers for Medicare \& Medicaid Services.

\section{Compliance with Ethical Standards:}

Conflict of Interest: The authors declare that they do not have a conflict of interest.

Disclaimer: The contents provided are solely the responsibility of the authors and do not necessarily represent the official views of HHS or any of its agencies.

\section{REFERENCES}

1. Goldberg DG, Beeson T, Kuzel AJ, Love LE, Carver MC. Team-based care: A critical element of primary care practice transformation. Popul Health Manag. 2013;16:150-6.

2. Hogg W, Lemelin J, Dahrouge S, et al. Randomized controlled trial of Anticipatory and Preventive multidisciplinary Team Care: For complex patients in a community-based primary care setting. Can Fam Physician. 2009;55:e76-e85.

3. Jesmin S, Thind A, Sarma $\mathbf{S}$. Does team-based primary health care improve patients' perception of outcomes? Evidence from the 2007-08 Canadian Survey of Experiences with Primary Health. Health Policy. 2012;105:71-83.

4. Kanter M, Martinez O, Lindsay G, Andrews K, Denver C. Proactive office encounter: A systematic approach to preventive and chronic care at every patient encounter. Perm J. 2010;14:38-43.

5. McLean DL, McAlister FA, Johnson JA, et al. A randomized trial of the effect of community pharmacist and nurse care on improving blood pressure management in patients with diabetes mellitus: Study of cardiovascular risk intervention by pharmacists-hypertension (scriphtn). Arch Intern Med. 2008;168:2355-61.

6. Pape GA, Hunt JS, Butler KL, et al. Team-based care approach to cholesterol management in diabetes mellitus: Two-year cluster randomized controlled trial. Arch Intern Med. 2011;171:1480-6.

7. Rosser WW, Colwill JM, Kasperski J, Wilson L. Progress of Ontario's Family Health Team Model: A patient-centered medical home. Ann Fam Med. 2011;9:165-71.

8. Fast Company. Kaiser Permanente designed a health center that puts patients first. Available at: https://www.fastcompany.com/3057404/ world-changing-ideas/kaiser-permanente-designed-a-health-centerthat-puts-patients-first.

9. U.S. Department of Veterans Affairs. PACT Space Module design guide. 2015.

10. Rosen MA, DiazGranados D, Dietz AS, et al. Teamwork in healthcare: Key discoveries enabling safer, high-quality care. Am Psychol. 2018;73:433.

11. Gunn R, Davis MM, Hall J, et al. Designing clinical space for the delivery of integrated behavioral health and primary care. J Am Board Fam Med. 2015;28 Suppl 1:S52-62.

12. Stroebel RJ, Obeidat B, Lim L, Mitchell JD, Jasperson DB, Zimring C The impact of clinic design on teamwork development in primary care. Health Care Manage Rev. 2019. doi: https://doi.org/10.1097/HMR. 0000000000000259

13. Bernstein ES, Turban $\mathbf{S}$. The impact of the "open" workspace on human collaboration. Phil Trans R Soc Lond B Biol Sci. 2018;373:20170239.

14. Bernstein ES. The transparency paradox: A role for privacy in organizational learning and operational control. Adm Sci Q. 2012; 57(2):181-216.

15. Morgan S, Pullon S, McKinlay E. Observation of interprofessional collaborative practice in primary care teams: An integrative literature review. Int J Nurs Stud. 2015;52:1217-30.

16. Cai $\mathbf{H}$, Zimring $\mathbf{C}$. Out of sight, out of reach: Correlating spatial metrics of nurse station typology with nurses' communication and co-awareness in 
an intensive care unit. Proceedings of the Eighth International Space Syntax Symposium. 2012;8039: 1-16

17. Gharaveis A, Hamilton DK, Pati D. The impact of environmental design on teamwork and communication in healthcare facilities: A systematic literature review. HERD. 2018;11:119-37.

18. Stock R, Mahoney E, Carney PA. Measuring team development in clinical care settings. Fam Med. 2013;45(10):691-700.

19. Lim L, Kim M, Zimring C. Measuring interpersonal visual relationships in healthcare facilities: The Agent Visibility Model and SAVisualPower tool. HERD. 2019;12:203-16.

20. DuBose J, Lim L, Savitsky M. Making sense of behavior mapping in facility planning and design. Presentation at Healthcare Design Conference. Houston. 2016.
21. Pullon S, Morgan S, Macdonald L, McKinlay E, Gray B. Observation of interprofessional collaboration in primary care practice: A multiple case study. J Interprof Care. 2016;30:787-94.

22. Patterson BJ, Solimeo SL, Stewart KR, Rosenthal GE, Kaboli PJ, Lund BC. Perceptions of pharmacists' integration into patient-centered medical home teams. Res Social Adm Pharm. 2015;11:85-95.

Publisher's Note Springer Nature remains neutral with regard to jurisdictional claims in published maps and institutional affiliations. 